\title{
Research Priorities for HIV/M. tuberculosis Co-Infection
}

María Alejandra Alvarez ${ }^{1,}$, Patricia Arbelaez ${ }^{2}$, Francisco Ignacio Bastos ${ }^{3}$, Ben Berkhout ${ }^{4}$, Basudev Bhattacharya ${ }^{5}$, Gennady Bocharov ${ }^{6}$, Valery Chereshnev ${ }^{7}$, Paloma Cuchí $^{8}$, Martin Däumer ${ }^{9}$, Olga Demikhova ${ }^{10, \S}$, Knut Feldmann ${ }^{11}$, Luis F. García ${ }^{2, \S}$, Claudia Giehl ${ }^{12, \S, \not}$, Akash Gulalia ${ }^{13}$, Beate Kampmann ${ }^{14}$, Eduard Karamov ${ }^{15,8}$, Poloko Kebaabetswe ${ }^{16}$, Mikhail Kiselev ${ }^{17}$, Anne-Laure Knellwolf ${ }^{18,}$, Afranio L. Kritski ${ }^{19}$, Christoph Lange ${ }^{20}$, Cecilio López-Galíndez ${ }^{21}$, Albert Makone ${ }^{22}$, Anandi Martin ${ }^{23,}$, Harriet Mayanja-Kizza ${ }^{24}$, Ruth McNerney ${ }^{25}$, Andreas Meyerhans ${ }^{*}, 26, \$, \pi$, Giovanni Battista Migliori ${ }^{27}$, Mariza G. Morgado ${ }^{3,8}$, Jean Nachega ${ }^{28}$, Bagrey Ngwira $^{29}$, Michal Odermarsky ${ }^{30,8}$, Martin Ota ${ }^{31}$, Juan Carlos Palomino ${ }^{23,8}$, Gaby E. Pfyffer ${ }^{32}$, Wolfgang Preiser ${ }^{33,8}$, Viviana Ritacco ${ }^{1,}$, Jaime Robledo ${ }^{34}$, Camilla Rodrigues $^{35}$, Horacio Salomón ${ }^{36}$, Sofia Samper ${ }^{37}$, Jorge Sanchez ${ }^{38}$, Martina Sester ${ }^{*}, 39, \$, \uparrow$, Pradeep Seth ${ }^{40, \$}$, Boniswa Seti ${ }^{41}$, Igor Sidorovich $^{42}$, Rupak Singla ${ }^{43}$, Laure Sonnier $^{30,}$, Carlo Torti $^{44}$, Enrico Tortoli ${ }^{45,}$, , Wim Vandevelde ${ }^{3,0},{ }^{\S}$ Stefano Vella ${ }^{18}$, Valdilea Veloso ${ }^{3,8}$, Hagen von Briesen ${ }^{46, \S}$, Kamini Walia ${ }^{47}$, Gerhard Walzl $^{33, \S}$ and Claire Wingfield ${ }^{48}$

${ }^{1}$ CONICET, Argentina; ${ }^{2}$ Universidad de Antioquia, Colombia $;{ }^{3}$ FIOCRUZ, Brazil; ${ }^{4}$ University of Amsterdam, AMC, The Netherlands; ${ }^{5}$ Institute of Post-graduate Medical Education, India; ${ }^{6}$ Institute of Numerical Mathematics, Russian Academy of Sciences, Russia; ${ }^{7}$ Institute of Immunology and Physiology, Russian Academy of Sciences, Russia; ${ }^{8}$ UNAIDS, Switzerland; ${ }^{9}$ Institute of Immunology and Genetics, Germany; ${ }^{10}$ Central Research Institute of Tuberculosis, Russian Academy of Medical Sciences, Russia; ${ }^{11}$ Kuratorium Tuberkulose in der Welt e.V., Germany; ${ }^{12}$ European Research \& Project Office, Germany; ${ }^{13}$ BPS Women University, India; ${ }^{14}$ Imperial College London, UK, ${ }^{15}$ D.I. Ivanovsky Institute of Virology, Russian Academy of Medical Sciences, Russia; ${ }^{16}$ BOTUSA, Botswana: ${ }^{17}$ Ministry of Health and Social Development of the Russian Federation, Russia; ${ }^{18}$ Istituto Superiore di Sanitá, Italy; ${ }^{19}$ Federal University of Rio de Janeiro, Brazil; ${ }^{20}$ Research Center Borstel, Germany; ${ }^{21}$ Instituto de Salud Carlos III, Spain; ${ }^{22}$ Community Working Group On Health, Zimbabwe; ${ }^{23}$ Institute of Tropical Medicine, Belgium; ${ }^{24}$ College of Health Sciences, Makerere University, Uganda $;{ }^{25}$ London School of Hygiene \& Tropical Medicine, UK; ${ }^{26}$ Saarland University, Germany \& ICREA and University Pompeu Fabra, Spain; ${ }^{27}$ Fondazione Salvatore Maugeri, Italy; ${ }^{28}$ Johns Hopkins University, USA; ${ }^{29}$ Karonga Prevention Study, Malawi; ${ }^{30} E A T G$, Belgium; ${ }^{31}$ MRC, The Gambia; ${ }^{32}$ Luzerne General Hospital, Switzerland; ${ }^{33}$ Stellenbosch University, South Africa; ${ }^{34}$ Corporación para Investigaciones Biológicas, CIB-UPB, Colombia; ${ }^{35}$ Hinduja National Hospital, India; ${ }^{36}$ Argentinean National Reference Center for AIDS, Argentina; ${ }^{37}$ Instituto Aragonés de Ciencias de la Salud (I+CS), Spain; ${ }^{38}$ Asociacion Civil Impacta, Salud y Educacion, Peru; ${ }^{39}$ Saarland University, Germany; ${ }^{40}$ Seth Research Foundation, India; ${ }^{41}$ AIDS \& RIGHTS Alliance for Southern Africa, South Africa; ${ }^{42}$ SRC Institute of Immunology, Russia; ${ }^{43}$ L.R.S. Institute of Tuberculosis \& Respiratory Diseases, India; ${ }^{44}$ University of Brescia, Italy; ${ }^{45}$ Careggi University Hospital, Italy; ${ }^{46}$ Fraunhofer IBMT, Germany; ${ }^{47}$ Indian Council of Medical Research, India; ${ }^{48}$ Treatment Action Group, USA

\begin{abstract}
This document summarizes priority areas for joint research and concerted actions to counteract the public health threat of AIDS/TB as identified within the European Support Action "EUCO-Net" funded under the $7^{\text {th }}$ Framework Programme of the European Commission.
\end{abstract}

Keywords: Research priorities, pathogenesis, HIV/M. tuberculosis co-infection, treatment, prevention, diagnostics, EUCO-Net.

\footnotetext{
*Address correspondence to these authors at the Department of Transplant and Infection Immunology, Institute of Virology, Saarland University, 66421 Homburg, Germany; Tel: 0049-6841-16-23557; Fax: 0049-6841-1621347; E-mail: martina.sester@uks.eu or Infection Biology Laboratory, Department of Experimental and Health Sciences, University Pompeu Fabra, Barcelona, Spain; Tel: 0034-933-160-831;

E-mail: andreas.meyerhans@upf.edu

${ }^{\S}$ Member of EUCO-Net Core Consortium, ${ }^{\circ}$ Coordination, ${ }^{\circledR}$ Management.
}

\section{INTRODUCTION}

Key WHO policy documents have identified priorities for handling HIV/AIDS and TB. On the HIV/AIDS side, the document is aimed at pursuing the goal of "universal access to comprehensive prevention programs, treatment, care and support" by the year 2010 [1]. On the TB side, the "Stop TB Strategy", wishes to ensure universal access to high-quality 
diagnosis and patient-centred treatment for all TB patients (including those co-infected with HIV and harbouring multidrug resistant (MDR) and extensively drug resistant (XDR) TB strains), and to support the development of new and effective tools to prevent, detect and treat TB in line with the "Global Plan to Stop TB" [2, 3]. Furthermore, the "Interim Policy on collaborative TB/HIV activities" [4], starting from the principle "Two diseases, one patient", set out simultaneous patient-focused care delivery, and emphasized the necessity of implementing collaborative activities in addition to existing global strategies. However, despite this commonly agreed clear policy guidance and understanding of the need for concerted action at the international as well as at the national level, key challenges remain [5]. In addition, an integrated response to the coepidemic has yet to be realized and joint HIV/MTB activities are severely under-funded.

The EUCO-Net core consortium convened a panel of 60 experts to generate this roadmap that identifies global research needs in the field of AIDS and TB, and HIV/MTB co-infection. The expert team included representatives of scientists, health care professionals, policymakers, activists and people living with HIV/TB. Although considered of high importance, the EUCO-Net group has not developed any recommendations for development of vaccines and new drugs since these areas have been receiving by far the most funding throughout FP6 and the first three years of FP7 [6] or been part of the recent FP7 calls (see supplementary document on AIDS/TB funding by C. Giehl). The proposed priority areas for joint AIDS/TB research and concerted actions below are divided into basic sciences, clinical research, diagnostics as well as training and networking.

\section{PROPOSAL OF TOPICS FOR JOINT AIDS/TB RESEARCH AND CONCERTED ACTION}

\section{Basic Sciences}

Since the first description of HIV in 1983 as the causative agent of the acquired immunodeficiency syndrome, there has been an impressive progress in the understanding of HIV biology, its interaction with the infected host and a concomitant extension of therapy options. This was possible due to existing funding, which in turn was made available at least in part by strong activist groups that have forced societies to booster their research activities in this area. The same does not hold for TB. With the BCG vaccine available that at least prevents disseminated TB and TB meningitis in children, and the possibility to cure TB with antibiotic treatment, TB research has been marginal for decades. As a consequence, sensitive diagnostic tests and therapeutic drugs against HIV and TB are of tremendously uneven availability today. Given the rise of TB due to the HIV pandemic and the limited options to manage MDR- and XDR-TB, there are strong accumulated needs to improve (i) the mechanistic understanding of $\mathrm{TB}$ pathogenesis especially in the context of an HIV infection, (ii) the therapy options and procedures for their implementation, and (iii) the diagnostic tests predictive for latent infection and active disease.

Two topics in basic science were identified that deserve further attention by concerted research activities and to which European scientists can substantially contribute. Topic
1 (see column) aims to understand in greater detail the HIV/MTB interactions within an infected host that lead to enhanced disease progression. A key element here is the formation of MTB-induced granuloma, its role in MTB containment, latency and release [7, 8]. How are these individual functions regulated and how influenced by HIV and the antiviral immune response? A consortium comprising of clinicians, pathologists, immunologists, microbiologists and virologists with access to up-to-date life imaging tools and relevant animal models could have a substantial impact on dissecting the cellular events that are at the heart of the disease. Besides the increase in basic knowledge, novel therapeutic strategies on how to contain MTB in latency or how to eliminate MTB kept latent are likely benefits from such a basic research approach.

Topic 2 continues along the significant progress made in understanding the development of HIV drug resistance [9, 10]. It aims to transfer this progress to the TB world and to combine it with TB-specific problems of drug resistance. Within the bacterial world, MTB seems unusual as it acquires much of its drug resistance by genetic mutations within the bacterial genome while for other bacteria, plasmid exchange is more common. In this respect MTB behaves like viruses for which drug resistance is associated with the outgrowth of respective virus mutants. Interestingly, it was first recognized in $\mathrm{TB}$ treatment that combination therapies are more successful than monotherapies to obtain long term clinical benefits [11-14]. Today the combination of drugs targeting different functions of the same virus is common practise.

The idea behind Topic $\mathbf{2}$ is simple: by understanding drug resistance pathways, defining microbial costs associated with resistance, considering immune and nutritional status, and incorporating the knowledge on drug functions and interactions, one should be able predict optimal therapy strategies especially when the first line drugs are failing. A consortium incorporating clinicians, basic researchers, drug designers and bioinformaticians should be able to significantly improve HIV/MTB management and help to reduce the frightening burden of multi-drug resistance worldwide.

Topic 1: Understanding MTB pathogenesis in HIV infected individuals by identification of microbial virulence and host factors

Aim: The aim is to understand both microbial and host mechanisms that contribute to different dynamics and clinical phenotypes of HIV/MTB co-infection. This will be achieved through highly standardized cohort studies, and the development and use of pre-clinical model systems (cell based or animal models).

Expected Impact: Main goals should include prediction of TB disease progression across different stages of the HIV related disease. Specifically, the precise knowledge on the pathogen interaction at the molecular and cellular level in the context of HIV/MTB co-infection and disease will contribute to the identification of new potential therapeutic targets.

Justification: HIV/MTB co-infection and the mutual interaction of the two pathogens is complex and poorly understood.

Topic 2: Individualized treatment approach for TB and HIV positive individuals based on drug resistance and therapeutic drug monitoring

Aim: The aim is to optimally tailor treatment in HIV/MTB co-infected patients through therapeutic drug monitoring and the development of 
algorithms that predict microbial drug resistance. Based on the substantial knowledge on genotype, phenotype and clinical resistance in the context of HIV, a similar strategy will be developed for infection with MTB. Main goals should include the identification of new drug resistance markers, mechanisms of drug resistance and evolution of microbial fitness. Systematic sample collection for host genetic analysis should also be performed.

Expected Impact: This will lead to an individualization of treatment as well as prevention of therapy failure and spread of drug resistant HIV/MTB variants.

Justification: Prediction of treatment efficacy, treatment success and therapy failure is not possible.

\section{Clinical Sciences}

The interplay between the two epidemics is clearly illustrated by the situation in the sub-Saharan region of Africa, which harbours $79 \%$ of all HIV/MTB co-infected individuals [15]. Among other regions such as Russia, India, or South America, the Southern African countries are particularly hard hit as is documented by a rate of TB in excess of 2.000 per 100.000 population in a Cape Town (South Africa) informal settlement, which is largely driven by the high prevalence of undiagnosed TB [16]. Current WHO endorsed strategies, including the directly observed therapy-short course (DOTS) are clearly not sufficient to control the HIV and TB epidemic, although failed implementation of these measures could also be to blame [17].

While the risk of TB during the HIV sero-conversion period seems unaltered, this risk raises three fold in the first two years after sero-conversion [17] and there is an increased risk of recurrent TB [18], especially as the CD4 counts decline. Molecular epidemiological data demonstrated that most recurrent episodes may be the result of exogenous reinfections rather than reactivation of latent infection in HIVuninfected [19] and in HIV-infected patients [18].

Multiple studies have shown a substantial survival benefit from antiretroviral therapy but active TB still occurs commonly before the diagnosis of HIV infection [20]. Many patients die of TB shortly after the initiation of antiretroviral therapy [21], there are numerous drug interactions between anti-tuberculous and antiretroviral drugs [22] and the pharmacokinetics of the drugs in adults and particularly in children are incompletely understood [23].

The effect of HIV on MDR and XDR-TB is of concern as the large population of HIV-infected susceptible hosts with poor TB treatment success rates, the lack of infection control measures in resource-poor settings, inadequate drugsensitivity testing and implementation of MDR-TB treatment programs could combine to lead to a MDR-TB and XDR-TB epidemic of unmatched scale [24]. The key challenges in this research area are summarised in Topics 3-6.

Topic 3: TB control activities in HIV-positive individuals: Studies of HIV/AIDS cohorts

Aim: The aim is to integrate existing and future cohort studies involving HIV-infected patients living in areas with high incidence of TB to increase statistical power. Main goals should be: improving diagnosis, prognosis, MTB infection control, prevention and treatment of TB, understanding the pathogenesis of this co-infection, especially immunopathogenesis, including IRIS and associated (predictive) factors increasing the understanding of causes of early mortality, outcomes of antiretroviral treatment (ART) in the context of hepatitis co-infection, and efficacy of TB preventive treatment. This network should build a multidisciplinary collaboration, bringing together investigators with different expertises from different countries. Inclusion of patients coinfected with hepatitis, adolescents and children will be considered an asset.

Expected Impact: A large cohort with harmonized and synchronized databases, sample collections and documentation from different health units in countries with high HIV/MTB co-infection will provide adequate statistical power for several retrospective and prospective analyses. Data using single cohorts or single countries can often not be evaluated and combining cohorts will be more cost effective than small and unpowered cohorts.

Justification: MTB and HIV co-infection is a major public health problem in many parts of the world, especially in the developing countries. Some questions can only be answered with large cohorts. However, funds available to these studies are very limited in countries highly affected by HIV-TB. This cohort will bring together investigators with different expertises from different countries and will also enhance the transfer of this expertise among them.

Topic 4: Treatment and Prevention of TB in HIV-positive individuals: Multicenter Clinical Trial Network on HIV/TB

Aim: The aim is to evaluate the interaction of ART and currently available TB drugs, new TB drugs, and different regimens for treatment and prevention of TB in HIV-positive patients with or without ART. The trial will also be designed to evaluate new treatment regimens for MDR and XDR TB among HIV co-infected patients. This will also include the evaluation of pharmacokinetics and pharmacosurveillance of anti TB treatment in the context of ART.

Expected Impact: This project will lead to an increased number of safe and potent drug regimens for treatment and prevention of TB in different settings of HIV infection according to different CD4 counts and/or different epidemiological situations. In addition, alternative safe drugs for the treatment of MDR and XDR-TB will be available to treat HIVinfected patients. This will increase our knowledge on pharmacokinetics in different settings of HIV/MTB co-infected individuals.

Justification: Sample sizes are often inadequate in particular for less common outcomes such as XDR and primary MDR. Clinical trials often require large numbers and a long time to complete each study and results may not readily be applied in different parts of the world with different TB dynamics, and strains and drug sensitivity patterns.

Topic 5: Study of pharmacokinetics of antiretroviral and anti-MTB drugs in relation to clinical and pharmacogenomic characteristics in children with HIV/MTB co-infection

Aim: The aim is to optimally define the drug dosages of anti-MTB and antiretroviral drugs in paediatric patients, since we already know that dosage regimens differ between children and adults when it comes to either HIV or TB treatment. Possible pharmacogenetic determinants of plasma drug levels will be identified with the final aim of individualizing drug dosage. Research should include the clinical follow-up for evaluation of treatment outcome in relation to plasma drug concentrations.

Expected Impact: This will lead to significant improvement in the therapeutic management of children infected by HIV/MTB.

Justification: Therapy of HIV/MTB co-infection in children is an area which is still poorly investigated.

Topic 6: Prevention of early mortality due to TB in HIV-positive individuals initiating ART in geographic regions of high TBincidence

Aim: The aim is the reduction in mortality due to TB in the first months following the initiation of ART in HIV-seropositive individuals. This study will be performed in different geographic areas with a high incidence of TB, e.g. India, Latin America, Sub-Saharan Africa, or Russia, where the lethality of TB is excessively high in the period following the initiation of ART. Possible geographic variations due to host and pathogen factors, different patterns of transmission as well as 
differences in treatment regimens should be elaborated. Main goals are the identification of risk factors for the TB-induced immune reconstitution inflammatory syndrome (IRIS) including markers of immune activation, genetic determinations and variables related to HIV and MTB-infection. Approaches should also include an interventional trial to administer anti-inflammatory medications in parallel to the initiation of ART to identify whether such an intervention is able to reduce early TB-related mortality following the initiation of ART in HIV-seropositive individuals. A comprehensive training scheme (including training on flow cytometry, cryopreservation, and molecular genetics etc) should be developed and implemented, and region-specific recommendations for treatment algorithms for IRIS in different geographical areas should be developed.

Expected impact: An increased knowledge on the effect of early ART will lead to a reduction in early mortality due to TB in HIV-infected individuals in countries of high TB incidence and will increase therapeutic options

Justification: TB is currently the leading cause of death among infectious diseases in HIV-infected individuals worldwide. The TBrelated mortality is excessively high in the first months following the initiation of ART. In this period, TB-related IRIS is significantly contributing to morbidity and mortality of HIV-seropositive individuals.

\section{Diagnostics}

Because TB is a treatable disease in principle, and HIV progression can largely be slowed down by antiretroviral drugs, rapid and efficient diagnosis of both infections is of prime importance in health care. With respect to HIV infection, very sensitive and specific diagnostic assays are available. They rely on the detection of specific antibodies, measurements of viral load and CD4 T-cell counts. Tests for accurate determination of these parameters do exist, although their wide-spread availability is largely restricted to industrialized countries [25]. In contrast, the diagnosis of a latent infection or an active TB disease is more demanding. This is particularly evident in HIV-infected individuals, where TB frequently manifests as an extrapulmonary disease with an atypical chest x-ray and a lower concentration of bacteria in sputum [26]. This leads to a considerable decrease in the sensitivity of most assays that rely on the detection of bacteria such as microscopy, culture or nucleic acid amplification. More sensitive rapid tests are urgently needed to improve case finding and access treatment. Novel technologies are required that can be used at point of care, or as screening devices in the community [6]. As a further advance, indirect measures of an infection such as the detection of components of adaptive immunity may be used [27-30]. They have the additional advantage that they are independent from the recovery of samples from various sites of bacterial growth that are often inaccessible in a noninvasive manner. Up to now, however, new immune-based tests such as the T-SPOT.TB and QuantiFERON TB gold in tube cannot distinguish between latent infection and active disease, and other tests (i.e. the tuberculin skin test) are of low sensitivity or specificity [31-35]. Moreover, the currently available tests are not appropriate for HIV-infected patients in high burden settings due to high costs and are insufficiently evaluated to predict the risk for disease progression and treatment success. In addition, drug resistance is an increasingly recognised problem in HIVinfected patients and knowledge on the prevalence of resistance patterns is limited.

The diagnosis and management of TB in HIV/MTB coinfected children is another major challenge [36-39]. In the year 2000, around $11 \%$ of the global TB cases were estimated to occur in children. The highest incidence was observed in sub-Saharan Africa where it is fuelled by the HIV epidemic. While HIV-infected children have an increased risk to develop active TB after MTB exposure, they rarely develop sputum smear-positive $\mathrm{TB}$ and the standard tuberculin skin test to diagnose the infection is of low sensitivity [38]. Symptom-based diagnostic approaches are also of little help because differential diagnosis with respect to other HIV-related or general paediatric clinical conditions is difficult.

To address these key research challenges in diagnostics, Topics 7-10 were designed. This need complies with Rio Communities' Declaration at the 3rd Stop TB Partners Forum (March 2009) as well as with the outcomes of the conference on "Challenges for the future: research on HIV/AIDS, Malaria and Tuberculosis" which the EC convened in Brussels in November 2008 to update the European research priorities in the field [6]. The development of new diagnostic tools that can provide faster and more accurate TB diagnosis for smear positive, smear negative and drug resistant $\mathrm{TB}$, including diagnostics appropriate for children has been defined as an urgent need and should therefore be a clear priority in future research.

Topic 7: Diagnosis of latent MTB infection in HIV-infected patients

Aim: The aim is to correctly identify patients latently infected with MTB, to predict their risk of progression, and to rule out active TB in both HIV-infected adult and paediatric patients. Key biomarkers should be identified, validated and implemented to be used in a clinical setting.

Expected Impact: This monitoring technology should facilitate rapid targeting of chemoprophylaxis and therapy, and will enable to quantify the risk for progression and treatment success.

Justification: In HIV infection, latent TB can rapidly progress to active disease. The currently available tests are not appropriate for HIVinfected patients in high burden settings and fail to affect patient care.

Topic 8: Diagnosis of active TB in HIV-infected patients

Aim: The aim is to develop and validate improved rapid screening technologies for active TB in both HIV-infected adult and paediatric patients that are appropriate for use in TB-endemic countries and poor resource settings. Those tests could be based on a variety of biological samples.

Expected Impact: This should lead to an improved case finding, better treatment outcomes, reduced transmission, and better targeting of preventive therapy.

Justification: TB is a leading cause of death in patients infected with HIV. Current diagnostic tools miss more than $50 \%$ of TB cases in HIVinfected individuals.

Topic 9: Diagnosis of drug resistance in $\mathrm{HIV}$-infected patients

Aim: The aim is to develop and validate improved screening technologies to assess resistance to HIV and TB drugs in both HIVinfected adult and paediatric patients.

Expected Impact: This should lead to prediction of treatment failure and quantitation of treatment efficacy. This will reduce transmission and improve patient care. Knowledge on drug resistance patterns will also inform infection control policies.

Justification: Drug resistance is an increasingly recognised problem in $\mathrm{HIV}$-infected patients. We need to know the prevalence of resistance patterns in order to plan the subsequent therapeutic options in HIV/MTB co-infected patients. 
Topic 10: Diagnosis of active TB in children in the context of HIV

Aim: The aim is to develop and validate new approaches that identify active TB in the context of HIV in children.

Expected Impact: This should lead to the adequate treatment of TB in children and to the prevention of TB/HIV-related childhood death.

Justification: Children are a neglected and vulnerable population often not included in TB studies. The lack of microbiological confirmation and the different clinical presentation of TB in children as compared to adults require innovative diagnostic approaches.

\section{Training and Networking}

The "cursed duet" of AIDS/TB continues to have a dramatic epidemiologic impact in settings at high HIV and MTB burden, with potentialities for further worsening where MDR- and XDR-TB are present [3, 40], e.g. in several countries of the former Soviet Union that are the gate to Europe. Although excellent documents have been designed by the WHO and their partners $(2,4-6)$ from both the HIV/AIDS and TB side, much still needs to be done to transform these principles into programmatic action. The principle "Two diseases, one patient", presently representing the "politically correct" vision of the two interlinked pandemics is far from being implemented into practice. At the programmatic level, within Ministries of Health of the priority countries, the national $\mathrm{TB}$ and HIV/AIDS programmes have problems to transform formal contacts into effective collaboration. Funding is still a matter of competition and non-equity. At the scientific level, HIV/AIDS and TB communities are largely separate, including their meetings and conferences. Moreover, they do not yet speak the same language. Just as an example, while the TB community prefers using the term Treatment of Latent Tuberculosis Infection (TLTBI), the HIV/AIDS one uses the term Isoniazid Preventive Therapy (IPT) to mention the same intervention. At the clinical level, important gaps still exist in the two communities to manage the "other" disease affecting the "one patient".

A major investment in training and networking is necessary at all levels (programmatic, scientific, clinical, political as well as social, psychological, etc.), if we want the "politically correct" principles are really transformed into action and the "one patient" can, at end, increase his/her chances of survival, and, hopefully, of cure. The key challenges are summarized in Topics 11-13.

\section{Topic 11: Training for translational research on $\mathrm{HIV} / \mathrm{TB}$}

Aim: In order to bridge the gaps between HIV and TB communities in the EU and the different high-burden regions, a comprehensive modular training approach is needed, targeted at clinicians, public health practitioners, researchers (incl. social researchers), patient community, policy makers, laboratory personnel, civil society groups, the media, and others.

The modules should be developed using established means including task analysis, training needs, etc in order to be able to address the different needs of the target groups mentioned above, and should be easily applicable in different target regions.

Expected Impact: To reach commonly agreed guidelines, identify best practices in HIV and TB management and prevention in different sites \& settings, improve management of HIV/MTB co-infected patients, improve planning, implementation, and monitoring and evaluation of HIV/MTB co-infection programmes, and create a common base for enhanced collaborative research efforts.

Justification: HIV/MTB co-infection is a global problem; however, lack of human resources knowledgeable in both fields is a recognized weakness limiting effectiveness of existing strategies. To date only a few initiatives address these interdisciplinary training needs. This project is expected to improve the availability of a sufficient number of competent human resources for health research and management, thus contributing to better access to essential health care and achieving the health-related WHO Millennium Development Goals.

\section{Topic 12: European TB Network of Excellence}

Aim: While several HIV Networks of Excellence (NoE) are in place already, at the moment, no European TB NoE is being funded by the EC. Support shall be given to a solid and inclusive research network on TB. Main goals of a Pan-European TB Network should include improving diagnosis and prognosis of patients infected with MTB (including drugresistant $\mathrm{TB}$ strains), understanding mechanisms of latent MTB infection, monitoring of treatment success, advancing understanding of pathogenesis of the infection, and proposing new interventions for infection control. The Network should be able to efficiently coordinate multi-disciplinary efforts to improve diagnosis and treatment of TB infection in Europe, both in adults and children. The inclusion of HIV co-infection issues will be considered as an asset, and should be closely coordinated with existing NoEs in the area of HIV research. The proposed NoE should provide a rationally organised joint programme of activities to implement common research goals and strategies, common research platforms, infrastructures and technologies, as well as standardised methods and protocols. Integration activities will furthermore comprise joint multidisciplinary training schemes and a concrete dissemination plan. Active participation of SMEs and/or Product Development Public-Private Partnership organisations could lead to an increased impact of the research proposed.

Expected Impact: This Network of Excellence will integrate and synergize ongoing TB initiatives in Europe and help create a durable joint structure to promote stronger institutional integration between core partners of the network, with the aim of structuring and strengthening the European Research Area in the field.

Justification: While several HIV NoEs are in place already, at the moment, no European TB NoE is being funded by the EC. In recent years, European TB research has successfully started to self-organize, and valuable networks have been created. These activities should be integrated to allow for concerted action and enable the creation of durable structures.

\section{Topic 13: Global HIV/TB network}

Aim: The aim is to provide long-term sustainable interaction and coordination of efforts addressing HIV/MTB co-infection. This will be achieved by detailed mapping of current networks of HIV, TB, and HIV/MTB co-infection (what is there, what is lacking, how to synergize existing initiatives). Activities should include the establishment of a joint repository of knowledge, the creation of decision making models to aid national policy makers, the promotion of infection control measures for HIV and MTB infections, monitoring the implementation of existing HIV and TB guidelines, the implementation of a guideline quality control system, and assurance of fast integration of new developments into existing guidelines. A process to engage key stakeholders in consensus building should be established. Sustainable networking with research partners and other stakeholders in disease-endemic countries should be an essential part of the project, providing a sound basis for integrated global research efforts across disciplines and diseases. Child health and ageing aspects should be taken into consideration wherever appropriate. Target regions should include Africa, India, Latin America, Russia, and SE Asia.

Expected Impact: This global NoE will integrate HIV and TB research in Europe, and connect the EU scientific community with all major endemic regions as well as leading global initiatives. It will build capacities and create a common knowledge base for coordinated action. A wide range of stakeholders, including especially patient organisations, should be represented.

Justification: Final Report on Challenges for the Future meeting (Brussels, Nov 2008) is advocating networking and mutual opening up of research groups, as well as streamlining data collection. 


\section{CONCLUSIONS}

A number of topics for future EU calls were defined by an international team of HIV/TB experts which may help guiding decisions by the EU and other funding bodies. By funding EUCO-Net, the EU underlines their commitment in supporting the global health challenge posed by HIV/MTB co-infection. From the participants' side, the collaborative effort was a highly efficient and successful experience, demonstrating that the integration of experts with different scientific and cultural backgrounds can indeed lead to significant synergies. Solid interactions were already established and strengthened during the project's lifetime and complementarities among partners will facilitate future collaborative activities in the area of HIV/MTB co-infection.

\section{ACKNOWLEDGEMENT}

MS is funded by the EU; AM is funded by the EU and the Bill and Melinda Gates foundation. The EUCO-Net project leading to these results has received funding from the European Community's Seventh Framework Programme (FP7/2007-2013) under Grant Agreement n²23373.

\section{REFERENCES}

[1] WHO. HIV/AIDS programme: towards universal access 2010. Geneva 2006.

[2] Raviglione MC, Uplekar MW. WHO's new Stop TB Strategy. Lancet 2006; 367(9514): 952-955.

[3] Migliori GB, Loddenkemper R, Blasi F, Raviglione MC. 125 years after Robert Koch's discovery of the tubercle bacillus: the new XDR-TB threat. Is "science" enough to tackle the epidemic? Eur Respir J 2007; 29(3): 423-7.

[4] WHO. Interim Policy on collaborative TB/HIV activities. Geneva 2004.

[5] Sester M, Giehl C, McNerney R, et al. Challenges and perspectives for improved management of HIV/Mycobacterium tuberculosis coinfection. Eur Respir J 2010; 36(6): 1242-7.

[6] Lang H, Quaglio G, Olesen OF. Tuberculosis research in the European union: past achievements and future challenges. Tuberculosis (Edinb) 2010; 90(1): 1-6.

[7] Barry CE, 3rd, Boshoff HI, Dartois V, et al. The spectrum of latent tuberculosis: rethinking the biology and intervention strategies. Nat Rev Microbiol 2009; 7(12): 845-55.

[8] Russell DG, Cardona PJ, Kim MJ, Allain S, Altare F. Foamy macrophages and the progression of the human tuberculosis granuloma. Nat Immunol 2009; 10(9): 943-8.

[9] Rosen-Zvi M, Altmann A, Prosperi M, et al. Selecting anti-HIV therapies based on a variety of genomic and clinical factors. Bioinformatics 2008; 24(13): i399-406.

[10] Deforche K, Cozzi-Lepri A, Theys K, et al. Modelled in vivo HIV fitness under drug selective pressure and estimated genetic barrier towards resistance are predictive for virological response. Antivir Ther 2008; 13(3): 399-407.

[11] Canetti G, Grosset J. [Percentage of isoniazid-resistant and streptomycin-resistant variants in wild strains of Mycobacterium tuberculosis on Loewenstein-Jensen medium.]. Ann Inst Pasteur (Paris) 1961; 101:28-46.

[12] Cohn ML, Middlebrook G, Russell WF Jr. Combined drug treatment of tuberculosis. I. Prevention of emergence of mutant populations of tubercle bacilli resistant to both streptomycin and isoniazid in vitro. J Clin Invest 1959; 38(8): 1349-55.

[13] Mitchison DA. The diagnosis and therapy of tuberculosis during the past 100 years. Am J Respir Crit Care Med 2005; 171(7): 699706

[14] Fox W, Ellard GA, Mitchison DA. Studies on the treatment of tuberculosis undertaken by the British Medical Research Council tuberculosis units, 1946-1986, with relevant subsequent publications. Int J Tuberc Lung Dis 1999; 3(10 Suppl 2): S231-79.

[15] WHO. Global tuberculosis control: epidemiology, strategy, financing. Geneva: World Health Organisation 2009.
[16] Middelkoop K, Bekker LG, Myer L, Dawson R, Wood R. Rates of tuberculosis transmission to children and adolescents in a community with a high prevalence of HIV infection among adults. Clin Infect Dis 2008; 47(3): 349-55.

[17] Lawn SD, Bekker LG, Middelkoop K, Myer L, Wood R. Impact of HIV infection on the epidemiology of tuberculosis in a peri-urban community in South Africa: the need for age-specific interventions. Clin Infect Dis 2006; 42(7): 1040-7.

[18] Charalambous S, Grant AD, Moloi V, Warren R, Day JH, van Helden P, Hayes RJ, Fielding KL, De Cock KM, Chaisson RE, Churchyard GJ. Contribution of reinfection to recurrent tuberculosis in South African gold miners. Int J Tuberc Lung Dis 2008; 12(8): 942-8.

[19] van Rie A, Warren R, Richardson M, et al. Exogenous reinfection as a cause of recurrent tuberculosis after curative treatment. $\mathrm{N}$ Engl J Med 1999; 341(16): 1174-9.

[20] Muga R, Ferreros I, Langohr K, et al. Changes in the incidence of tuberculosis in a cohort of HIV-seroconverters before and after the introduction of HAART. AIDS 2007; 21(18): 2521-7.

[21] Lawn SD, Myer L, Bekker LG, Wood R. Tuberculosis-associated immune reconstitution disease: incidence, risk factors and impact in an antiretroviral treatment service in South Africa. AIDS 2007; 21(3): 335-41.

[22] Ma Z, Lienhardt C. Toward an optimized therapy for tuberculosis? Drugs in clinical trials and in preclinical development. Clin Chest Med 2009; 30(4): 755-68, ix.

[23] Donald PR, Diacon AH. The early bactericidal activity of antituberculosis drugs: a literature review. Tuberculosis (Edinb) 2008; 88(Suppl 1): S75-83.

[24] Andrews JR, Shah NS, Gandhi N, Moll T, Friedland G. Multidrugresistant and extensively drug-resistant tuberculosis: implications for the HIV epidemic and antiretroviral therapy rollout in South Africa. J Infect Dis 2007; 196(Suppl 3): S482-90.

[25] Drosten C, Panning M, Drexler JF, et al. Ultrasensitive monitoring of HIV-1 viral load by a low-cost real-time reverse transcriptionPCR assay with internal control for the 5 ' long terminal repeat domain. Clin Chem 2006; 52(7): 1258-66.

[26] Perkins MD, Cunningham J. Facing the crisis: Improving the diagnosis of tuberculosis in the HIV era. J Infect Dis 2007; 196(Suppl 1): S15-S27.

[27] del Corral $\mathrm{H}$, Paris SC, Marin ND, et al. IFNg response to Mycobacterium tuberculosis, risk of infection and disease in household contacts of tuberculosis patients in Colombia. PLoS One 2009; 12(4): e8259, doi:8210.1371/journal.pone.0008257.

[28] Bamford AR, Crook AM, Clark J, et al. Comparison of Interferongamma release assays and Tuberculin Skin Test in predicting active tuberculosis (TB) in children in the UK- a Paediatric TB Network Study. Arch Dis Child 2009.

[29] Sester M, Sester U, Clauer P, et al. Tuberculin skin testing underestimates a high prevalence of latent tuberculosis infection in hemodialysis patients. Kidney Int 2004; 65(5): 1826-34.

[30] Sester U, Junker H, Hodapp T, et al. Improved efficiency in detecting cellular immunity towards $M$. tuberculosis in patients receiving immunosuppressive drug therapy. Nephrol Dial Transplant 2006; 21: 3258-68.

[31] Pai M, Riley LW, Colford JM, Jr. Interferon-gamma assays in the immunodiagnosis of tuberculosis: a systematic review. Lancet Infect Dis 2004; 4(12): 761-76.

[32] Dinnes J, Deeks J, Kunst H, et al. A systematic review of rapid diagnostic tests for the detection of tuberculosis infection. Health Technol Assess 2007; 11(3): 1-196.

[33] Diel R, Loddenkemper R, Nienhaus A. Evidence based comparison of commercial interferon-gamma release assays for detecting active tuberculosis -- a meta-analysis. Chest 2009.

[34] Diel R, Goletti D, Ferrara G, et al. Interferon-gamma release assays for the diagnosis of latent Mycobacterium tuberculosis infection: a systematic review and meta-analysis. Eur Respir J 2011; 37(1): 8899.

[35] Sester M, Sotgiu G, Lange C, et al. Interferon- $\gamma$ release assays for the diagnosis of active tuberculosis: a systematic review and metaanalysis. Eur Respir J 2011; 37(1): 100-11.

[36] Newton SM, Brent AJ, Anderson S, Whittaker E, Kampmann B. Paediatric tuberculosis. Lancet Infect Dis 2008; 8(8): 498-510.

[37] Kampmann B, Tena-Coki G, Anderson S. Blood tests for diagnosis of tuberculosis. Lancet 2006; 368(9532): 282; author reply 282-3. 
[38] Marais BJ, Graham SM, Cotton MF, Beyers N. Diagnostic and management challenges for childhood tuberculosis in the era of HIV. J Infect Dis 2007; 196(Suppl 1): S76-S85.

[39] Kampmann B, Whittaker E, Williams A, et al. Interferon-gamma release assays do not identify more children with active tuberculosis than the tuberculin skin test. Eur Respir J 2009; 33(6): 1374-82.

[40] Sotgiu G, Ferrara G, Matteelli A, et al. Epidemiology and clinical management of XDR-TB: a systematic review by TBNET. Eur Respir J 2009; 33(4): 871-81.

Received: November 26, 2010

(C) Alvarez et al.; Licensee Bentham Open.

This is an open access article licensed under the terms of the Creative Commons Attribution Non-Commercial License (http://creativecommons.org/licenses/ by-nc/3.0/) which permits unrestricted, non-commercial use, distribution and reproduction in any medium, provided the work is properly cited. 